\title{
Performance Analysis of the V-BLAST Algorithm: An Analytical Approach
}

\author{
S. Loyka ${ }^{1}$, F. Gagnon ${ }^{2}$
}

\begin{abstract}
An analytical approach to the performance analysis of the V-BLAST algorithm is presented in this paper, which is based on the analytical model of the Gramm-Schmidt process. Closed-form analytical expressions of the vector signal at $i$-th processing step and its power are presented. A rigorous proof that the diversity order at $i$-th step (without optimal ordering) is $(n-m+i)$ is given. It is shown that the optimal ordering is based on the least correlation criterion and that the afterprocessing signal power is determined by the channel correlation matrices in a fashion similar to the channel capacity.
\end{abstract}

Index Terms-MIMO, V-BLAST, multi-antenna system, fading

\section{INTRODUCTION}

Information-theoretic considerations show that the multipleinput multiple-output (MIMO) communication architecture is able to provide extraordinary high spectral efficiencies in rich multipath environments, which are simply unattainable using conventional techniques [1-4]. Space-time coding and/or a special signal processing algorithm is to be implemented at the receiver in order to achieve at least part of the MIMO channel capacity. Diagonal Bell Labs Layered Space-Time (D-BLAST) algorithm has been proposed by Foschini for this purpose, which is capable of achieving a substantial part of the MIMO capacity [2]. However, a high complexity of the algorithm implementation is its substantial drawback. A simplified version of the BLAST algorithm is known as V-BLAST (vertical BLAST). It is capable of achieving high spectral efficiency while being relatively simple to implement [5].

Comprehensive evaluation of the system performance is required because the matrix wireless propagation channel may severely degrade the performance of this algorithm [68]. Some preliminary studies including asymptotic analysis and numerical Monte-Carlo simulations have been reported in [9]. While the numerical Monte-Carlo approach is useful from many viewpoints, the analytical approach provides deeper insight and comprehensive understanding of the key points in the algorithm operation.

In this paper, we develop a unified analytical approach to the analysis of the V-BLAST algorithm operation based on some general geometrical ideas. This approach is based on the closed-form analytical models of the key V-BLAST and

${ }^{1}$ School of Information Technology and Engineering (SITE)

University of Ottawa, 161 Louis Pasteur, Ottawa, Ontario, Canada

K1N 6N5 (e-mail: sergey.loyka@ieee.org)

${ }^{2}$ Department of Electrical Engineering, Ecole de Technologie Superieure

1100, Notre-Dame St. West, Montreal (Quebec), H3C 1K3, Canada associated system components - interference nulling from yet to be detected symbols (Gramm-Schmidt orthogonalization process), interference subtraction from already detected symbols, the optimal ordering procedure (based on the after processing SNR), optimal (maximum ratio or similar) combining, and a statistical (complex Gaussian) model of the matrix wireless propagation channel. In particular, we derive closed-form analytical expressions for the signal and noise vectors at each processing step for wireless channel with the general correlation matrix. Based on these results, we give a rigorous proof that the diversity order at the $\mathrm{i}$-th processing step is $n-m+i$ (where $n$ and $m$ are the number of $\mathrm{Rx}$ and $\mathrm{Tx}$ antennas correspondingly) for uncorrelated Rayleigh channel and if no optimal ordering is used. At the moment, we are not able to analyze analytically the diversity order when the optimal ordering is implemented, but numerical Monte-Carlo analysis shows that the effect of optimal ordering is approximately to increase the after processing SNR by few dBs rather than to increase the diversity order (as one would intuitively expect based on the selection combining argument). Note that the analysis results above allows one to find the outage curves (i.e., fade level versus outage probability) and, hence, to estimate the average bit error rate (BER), in some cases analytically as well.

\section{V-BLAST ALGORITHM}

The V-BLAST algorithm has been discussed in details elsewhere [5,9]. Here we describe its main points for completeness and in order to introduce notations. The main idea of the BLAST architecture is to split the information bit stream into several sub-streams and transmit them in parallel using a set of Tx antennas (the number of Tx antennas equals the number of sub-streams) at the same time and frequency. At the Rx side, each Rx antennas "sees" all the transmitted signals, which are mixed due to the nature of the wireless propagation channel. Using appropriate signal processing at the Rx side, these signals can be unmixed so that the matrix wireless channel is transformed into a set of virtual parallel independent channels (provided that mutltipath is rich enough).

The following basic assumptions are employed:

- The channel is random, quasistatic (i.e. fixed for every frame of information bits but varying from frame to frame), frequency independent (i.e., negligible delay spread) and with complex AWGN. 
- The Tx signal vector is comprised of individual symbol sub-streams. No space-time coding is employed.

- The noise vector is comprised of independent AWGN components with equal variance.

- The Tx signals, noise and channel gains are independent of each other

- Perfect channel knowledge is assumed to be available at the receiver.

- There is no performance degradation due to synchronization and timing errors.

The received signal vector $\mathbf{r}$ can be presented in the following complex baseband vector form [9]:

$$
\mathbf{r}=\mathbf{H q}+\mathrm{v}
$$

where $\mathbf{q}=\left[\begin{array}{lll}q_{1} & \ldots & q_{m}\end{array}\right]^{\mathrm{T}}$ is the transmitted symbol vector, $\mathbf{H}$ is the channel matrix (i.e., the matrix of complex transfer factors from each $\mathrm{Tx}$ to each $\mathrm{Rx}$ antenna), and $v=\left[\begin{array}{lll}v_{1} & \ldots & v_{n}\end{array}\right]^{\mathrm{T}}$ is the noise vector. Presenting the channel matrix in a column-wise way, $\mathbf{H}=\left[\begin{array}{lll}\mathbf{h}_{1} & \ldots & \mathbf{h}_{m}\end{array}\right]$, where $\mathbf{h}_{\mathrm{i}}$ is a column vector of transfer factors from i-th Tx antenna to all $\mathrm{Rx}$ antennas, the received signal can be presented as:

$$
\mathbf{r}=\sum_{i} \mathbf{h}_{i} q_{i}+\mathrm{v}
$$

The V-BLAST processing begins with the $1^{\text {st }}$ Tx symbol and proceeds in sequence to the $\mathrm{m}$-th symbol. When the optimal ordering procedure is employed, the Tx indexing is changed prior to the processing. The main steps of the V-BLAST processing (detection) algorithm are as follows [5,9]:

1. The interference cancellation step: at the i-th processing step (i.e., when the signal from the i-th transmitter is detected) the interference from the first i-1 transmitters can be subtracted based on the estimations of the Tx symbols (which are actually assumed to be error-free) and the knowledge of $\mathbf{H}$.

2. The interference nulling step: based on the knowledge of the channel matrix, the interference from yet-to-bedetected symbols can be nulled out using the GrammSchmidt orthogonalization process (applied to the column vectors of $\mathbf{H})$.

3. The optimal ordering procedure: the order of symbol processing is organized according to their after-processing SNRs in the decreasing order (i.e., the symbol with highest SNR is detected first).

\section{ANALYSIS OF THE V-BLAST ALGORITHM}

For the sake of notational simplicity, we first describe all the steps without the noise contribution $(v=0)$, which is added to the analysis later.
The interference cancellation step can be expressed mathematically in a straightforward way [9]. The received signal after the cancellation at the i-th step is:

$$
\mathbf{r}_{i}^{\prime}=\mathbf{r}-\sum_{j=1}^{i-1} \mathbf{h}_{j} \hat{q}_{j}
$$

where $\hat{q}_{j}$ are the estimations of the already-detected symbols.

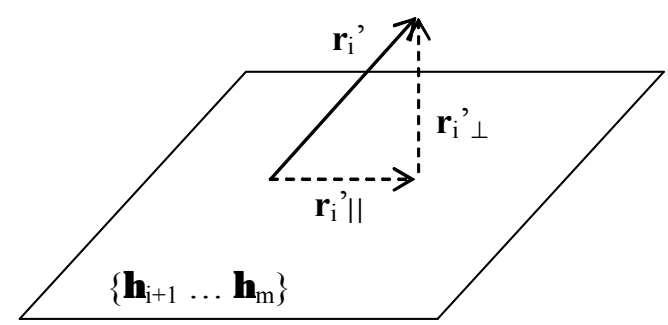

Figure 1. Geometric illustration of the interference nulling out step: the received vector (after the interference cancellation) is decomposed into orthogonal and parallel components with respect to the space spanned by $\left\{\begin{array}{lll}\eta_{i+1} & \ldots & \eta_{m}\end{array}\right\}$

The interference nulling step is based on the GrammSchmidt ortogonalization procedure, which builds a set of orthogonal vectors from a set of linearly-independent vectors. At this stage, we assume that $\mathbf{h}_{i}$ are linearly independent (otherwise the V-BLAST algorithm must be modified taking into account all the linearly dependent column vectors and decreasing the number of independent bit sub-streams). Using the closed form analytical expression for the Gramm-Schmidt process [10, p.214] and after some mathematical development (see Appendix for details), we arrive to the following expression of the received vector after interference nulling out and cancellation at the i-th step:

$$
\mathbf{r}_{i}^{\prime \prime}=\frac{q_{i}\left|\mathbf{h}_{i}\right|}{\left|\mathbf{R}^{[i+1, m]}\right|}\left|\begin{array}{cccc}
\eta_{i} & \eta_{i} \eta_{i+1} & \ldots & \eta_{i} \eta_{m} \\
\eta_{i+1} & & & \\
\ldots & & \mathbf{R}^{[i+1, m]} & \\
\eta_{m} & &
\end{array}\right|
$$

where $\left|\mathbf{h}_{i}\right|=\sqrt{\sum_{j}\left|h_{j i}\right|^{2}}, \quad||$ means determinant when applied to a matrix, $\eta_{i}=\mathbf{h}_{i} /\left|\mathbf{h}_{i}\right|, \eta_{i} \eta_{j}=\sum_{k=1}^{n} \eta_{k i} \eta_{k j} *$, * denotes complex conjugate, and $\mathbf{R}^{[i+1, m]}$ is the normalized channel correlation matrix built on $\left[\begin{array}{lll}\eta_{i+1} & \ldots & \eta_{m}\end{array}\right]$,

$$
\mathbf{R}^{[i+1, m]}=\left[\begin{array}{cccc}
1 & \eta_{i+1} \eta_{i+2} & \ldots & \eta_{i+1} \eta_{m} \\
\eta_{i+2} \eta_{i+1} . & 1 & \ldots & \eta_{i+2} \eta_{m} \\
\ldots & \ldots & \ldots & \ldots \\
\eta_{m} \eta_{i+1} & \eta_{m} \eta_{i+2} & \ldots & 1
\end{array}\right]
$$

The signal power is simply expressed as: 


$$
\left|\mathbf{r}_{i}\right|^{2}=\left|q_{i}\right|^{2}\left|\mathbf{h}_{i}\right|^{2} \frac{\left|\mathbf{R}^{[i, m]}\right|}{\left|\mathbf{R}^{[i+1, m]}\right|}
$$

From this result and using (11), it is straightforward to obtain a bit error rate. It is instructive to consider the case of $m=2$. At the first processing step one obtains:

$$
\left|\mathbf{r}_{1}\right|^{2}=\left|q_{1}\right|^{2}\left|\mathbf{h}_{1}\right|^{2}\left(1-\left|R_{12}\right|^{2}\right)
$$

where $R_{12}=\eta_{1} \eta_{2}$. Hence, the received power and, consequently, SNR, is determined by the total received power from the $1^{\text {st }} \mathrm{Tx}$ antenna (transmitting the $1^{\text {st }}$ bit stream), e.g. $\left|q_{1}\right|^{2}\left|\mathbf{h}_{1}\right|^{2}$, and by the normalized channel correlation coefficient $R_{12}$. We would like to emphasize the similarity of the results above to the analogous results on the MIMO channel capacity [6,7], which is also determined by the channel correlation matrix (especially for the case of $2 \times 2$ MIMO architecture, i.e. [11]). One could intuitively conclude from (7) that the diversity order is $n$ because of $\left|\mathbf{h}_{1}\right|^{2}$, which actually means $n$-th order maximum-ratio combining (MRC). However, as we prove later, the effect of the last factor in (7) is such that the actual diversity order is $n$ - 1 . Let us now consider the optimal ordering procedure. To separate the effect of the transmitted symbol power (i.e., $\left.\left|q_{1}\right|^{2}\right)$ and of the noise power from the effect of the propagation channel, we assume that all $\left|q_{i}\right|$ are equal (i.e., constant amplitude modulation) and all per-branch noise powers are also equal. If the 1-st Tx symbol is detected first, then the after-processing power is given by (7). If the 2-nd Tx symbol is detected first, then (7) should be changed to:

$$
\left|\mathbf{r}_{1}\right|^{2}=\left|q_{2}\right|^{2}\left|\mathbf{h}_{2}\right|^{2}\left(1-\left|R_{12}\right|^{2}\right)
$$

When the noise power is equal in all branches, the afterprocessing SNR is proportional to the received symbol power. Then the optimal ordering is to detect first the symbol with the highest $\left|\mathbf{h}_{i}\right|^{2}$, i.e. the same as for the selection combining. However, as we show later, this does not result in the increase of the diversity order (due to the last factor in (7) and(8)).

Let us consider the optimal ordering at the 1-st step for arbitrary $m$. When the i-th Tx symbol is detected first, the signal power after interference nulling out is:

$$
P_{i}=\left|q_{i}\right|^{2}\left|\mathbf{h}_{i}\right|^{2} \frac{|\mathbf{R}|}{\left|\mathbf{R}^{[i]}\right|}
$$

where $\mathbf{R}$ is the full correlation matrix (i.e., built on $\left.\left[\begin{array}{lll}\eta_{1} & \ldots & \eta_{m}\end{array}\right]\right)$ and $\mathbf{R}^{[i]}$ is the correlation matrix built on all column vectors except for $\eta_{i}$. Under the assumptions of equal $\left|q_{i}\right|$ and equal $\left|\mathbf{h}_{i}\right|$ (i.e., the same received power from every transmit antenna), the optimal ordering is to detect first the symbol with the smallest $\left|\mathbf{R}^{[i]}\right|$. In fact, this means that the overall correlation among $\left[\begin{array}{llll}\eta_{1}, \ldots & \eta_{i-1}, & \eta_{i+1}, \ldots & \eta_{m}\end{array}\right]$ must be highest and, consequently, the correlation between $\eta_{i}$ and $\left[\begin{array}{llll}\eta_{1}, \ldots & \eta_{i-1}, & \eta_{i+1}, \ldots & \eta_{m}\end{array}\right]$ must be the lowest. Thus, the best ordering is to detect first that symbol whose column propagation vector has lowest correlation with the other vectors.

Let us know consider the effect of the noise. Eq. 4 is generalized as follows:

$$
\mathbf{r}_{i} "=\mathbf{r}_{0, i} "+v_{i} "
$$

where $\mathbf{r}_{0, i}$ " is given by (4) and

$$
v_{i}^{\prime \prime}=\frac{v}{\left|\mathbf{R}^{[i+1, m]}\right|}\left|\begin{array}{cccc}
1 & \eta_{i+1} & \ldots & \eta_{m} \\
\eta_{i+1} & & & \\
\ldots & & \mathbf{R}^{[i+1, m]} & \\
\eta_{m} & &
\end{array}\right|
$$

Using (10), the after-processing noise power at i-th step can be simply expressed as:

$$
P_{v_{i}}=\left\langle\left|v_{i}\right|^{2}\right\rangle=(n-m+i) \sigma_{1}^{2}
$$

where $\sigma_{1}^{2}=\left\langle\left|v_{j}\right|^{2}\right\rangle$ is per-branch noise power before processing, and \langle\rangle is the expectation over noise voltage. Note that the after-processing noise power is less than the total noise power, which is $n \sigma_{1}^{2}$. This is the consequence of the orthogonal projection performed by the Gramm-Schmidt process (see Fig. 1). One also should note that the afterprocessing noise power increases with $i$ (step index), being the smallest in the $1^{\text {st }}$ step and the same as the total noise power in the last step. Geometrical interpretation of the noise transformation during the V-BLAST processing is the same as in Fig. 1.

\section{FAding OUtAGe CURVES AND DIVERSITY ORDER}

Based on the results above, let us know analyze the signal fading in the V-BLAST system. In particular, we consider the outage probabilities (i.e., the probability that the signal level is less than the specified value) and diversity order (i.e., the asymptotic slope of the outage probability curve).

We assume that the channel gains (i.e., the components of H) are i.i.d. complex Gaussians with zero mean and unit variance (i.e., we consider only the channel variation due to multipath and ignore the absolute propagation loss and largescale variation due to shadowing). First, we ignore the 
optimal ordering procedure and prove that the diversity order at the $i$-th step is $(n-m+i)$.

To demonstrate the main idea of the proof, let us consider first the case of $n=m=2$, i.e. $\mathbf{H}=\left[\begin{array}{ll}\mathbf{h}_{1} & \mathbf{h}_{2}\end{array}\right]$. To be specific, we assume that the 1-st Tx symbol is detected first. The interference nulling out can be expressed is a general matrix form:

$$
\mathbf{r}_{\perp}=Q \cdot \mathbf{r}
$$

where $Q$ is an orthogonal projection matrix, which projects $\mathbf{r}$ to the direction orthogonal to $\mathbf{h}_{2}$. Substituting (12) into (2), one obtains (since we are interested in the received signal power only, we ignore noise in this section):

$$
\mathbf{r}_{\perp}=q_{1} Q \cdot \mathbf{h}_{1}
$$

This means that the signal after interference nulling out is proportional to that part of $\mathbf{h}_{1}$ which is orthogonal to $\mathbf{h}_{2}$, see Fig. 2, and the signal power $\sim\left|\mathbf{h}_{1 \perp}\right|^{2}$.

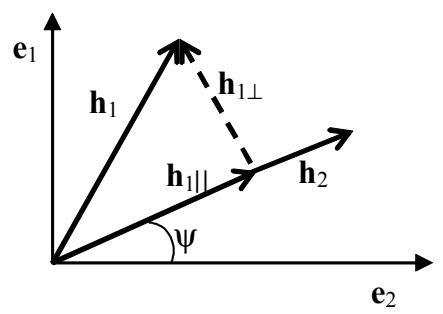

Figure 2. Geometrical representation of interference nulling out: decomposition of $\mathbf{h}_{1}$ into $\mathbf{h}_{1 \perp}$ and $\mathbf{h}_{1 \|}$.

But the vector magnitude is not affected by rotation on an arbitrary angle. We rotate $\left[\begin{array}{ll}\mathbf{h}_{1} & \mathbf{h}_{2}\end{array}\right]$ as a whole on angle $\psi$ so that $\mathbf{h}_{2}$ is parallel to $\mathbf{e}_{2}: h_{1,2}=0$. This can be expressed as:

$$
\tilde{\mathbf{h}}_{i}=A \cdot \mathbf{h}_{i}
$$

where $A$ is the rotation matrix, which satisfies to (preservation of length):

$$
A \cdot A^{+}=A^{+} \cdot A
$$

where "+" denotes conjugate transpose. Using (14), one obtains: $\left|\mathbf{h}_{1 \perp}\right|=\left|\tilde{h}_{1,1}\right|$. It is straightforward to show using (15) that the components of $\tilde{\mathbf{h}}_{1}$ has the same distribution as the components of $\mathbf{h}_{1}$ (note that $\psi$ is independent of $\mathbf{h}_{1}$ ), i.e. i.i.d. complex Gaussians with unit variance. Hence, $\left|\mathbf{h}_{1 \perp}\right|^{2}$ is chi-squared random variable with two degrees of freedom, $\left|\mathbf{h}_{1 \perp}\right|^{2} \sim \chi_{2}^{2}$. The same is true for the signal power. Thus, the diversity order in the 1-st step is one. The similar consideration for arbitrary $n$ leads to the conclusion that $\left|\mathbf{h}_{1 \perp}\right|^{2} \sim \chi_{2(n-1)}^{2} \quad$ (simply because $\mathbf{h}_{1 \perp}$ has $n-1$ non-zero components after rotation) and the diversity order is $(n-1)$.

The case of arbitrary $m$ is somewhat more complex however straightforward to consider in the similar way. First, we rotate the set $\left[\begin{array}{lll}\mathbf{h}_{1} & \ldots & \mathbf{h}_{m}\end{array}\right]$ as a whole so that $\mathbf{h}_{m}$ becomes parallel to $\mathbf{e}_{m}$. In the second rotation we keep $\mathbf{h}_{m}$ fixed (i.e., a rotation around $\mathbf{e}_{m}$ axis) and position $\mathbf{h}_{m-1}$ into $\left[\begin{array}{ll}\mathbf{e}_{m-1} & \mathbf{e}_{m}\end{array}\right]$ plane. The rotations are continued until $\mathbf{h}_{2}$ is positioned into $\left[\begin{array}{lll}\mathbf{e}_{2} \mathbf{e}_{3} & \ldots & \mathbf{e}_{m}\end{array}\right]$ hypeplane. After the rotation, $\mathbf{h}_{1 \perp}$ has $(n-m+1)$ non-zero components. Every such rotation preserves the distribution of the components. Hence, $\left|\mathbf{h}_{1 \perp}\right|^{2} \sim \chi_{2(n-m+1)}^{2}$ and the diversity order is $(n-m+1)$. Similar consideration for the i-th step leads to the conclusion that $\left|\mathbf{h}_{i \perp}\right|^{2} \sim \chi_{2(n-m+i)}^{2}$ and the diversity order is $(n-m+i)$. Note that the lowest diversity order is at the 1-st step and the highest is at the last (i.e., $n$ ). When $n=m$, no diversity is obtained at the 1-st step.

Unfortunately, we are not able at the moment to analyze analytically the optimal ordering procedure due to mathematical complications. Thus, we use numerical MonteCarlo simulations. First, the V-BLAST algorithm outage curves have been simulated without the optimal ordering. No difference has been observed between the analytical results above and the Monte-Carlo simulations (thus, the results are not shown here), which validates the analytical results. Secondly, the V-BLAST outage curves have been simulated with the optimal ordering procedure. Some of the results are presented in Fig. 3-5. They demonstrate that the effect of the optimal ordering for a moderate number of antennas is to increase signal power (and SNR) rather than to increase the diversity order. However, as shown on Fig. 5, it is difficult to observe this tendency for outage probabilities higher that approximately $10^{-4}$ when $n=4$. The $1^{\text {st }}$ and $2^{\text {nd }}$ step curves are mixed in this region.

\section{CONCLUSIONS}

Using a closed-from model of the Gramm-Schmidt process, we have developed an analytical approach to the performance analysis of the V-BLAST algorithm. In particular, closed-form analytical expressions have been presented for the signal and noise vectors at $i$-th processing step. The after-processing signal power is determined by the channel instantaneous correlation matrices (in the same fashion as the channel capacity is). The optimal ordering is proved to be equivalent to the least correlation criterion.

Performing the statistical analysis analytically for Rayleigh uncorrelated channel, we have proved that the diversity order 


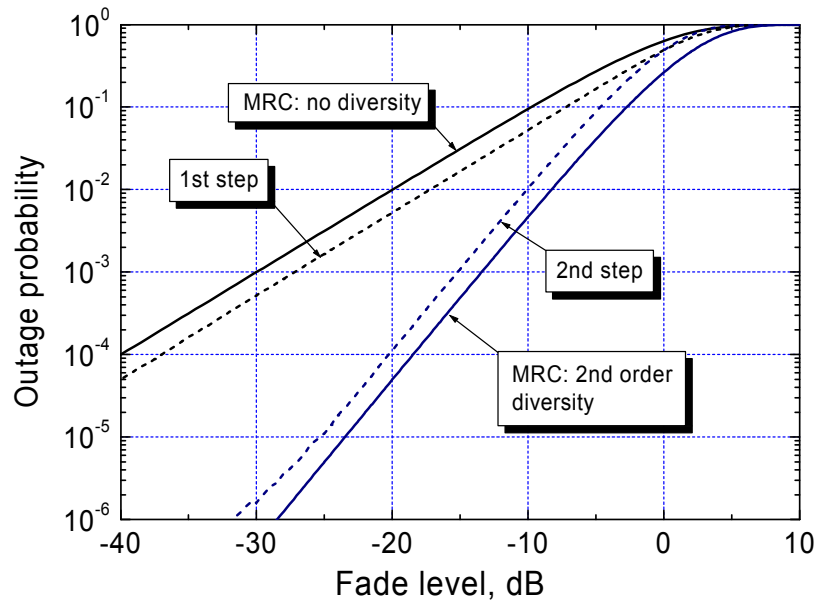

Figure 3. Outage probability curves of the V-BLAST algorithm for $\mathrm{n}=2$.

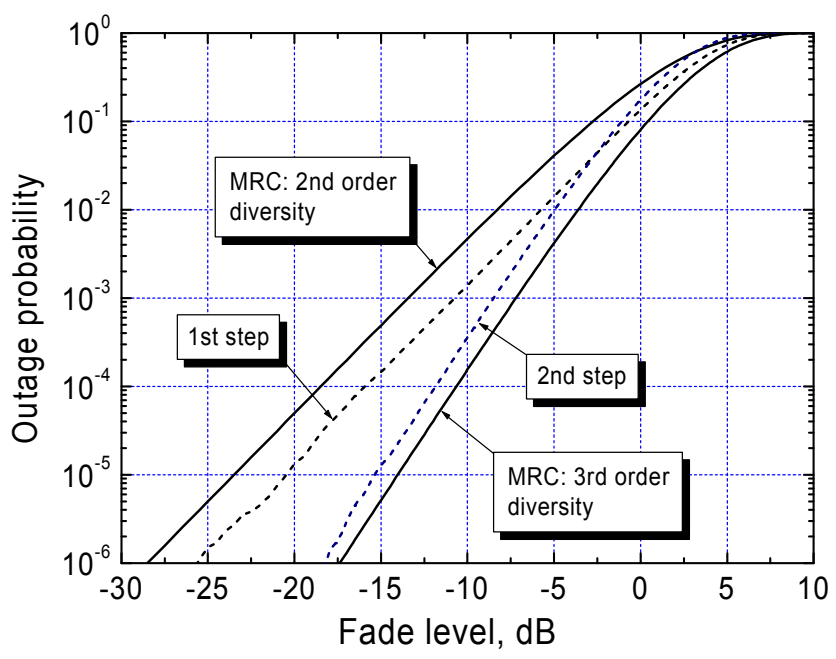

Figure 4. Outage probability curves of the V-BLAST algorithm for $n=3$.

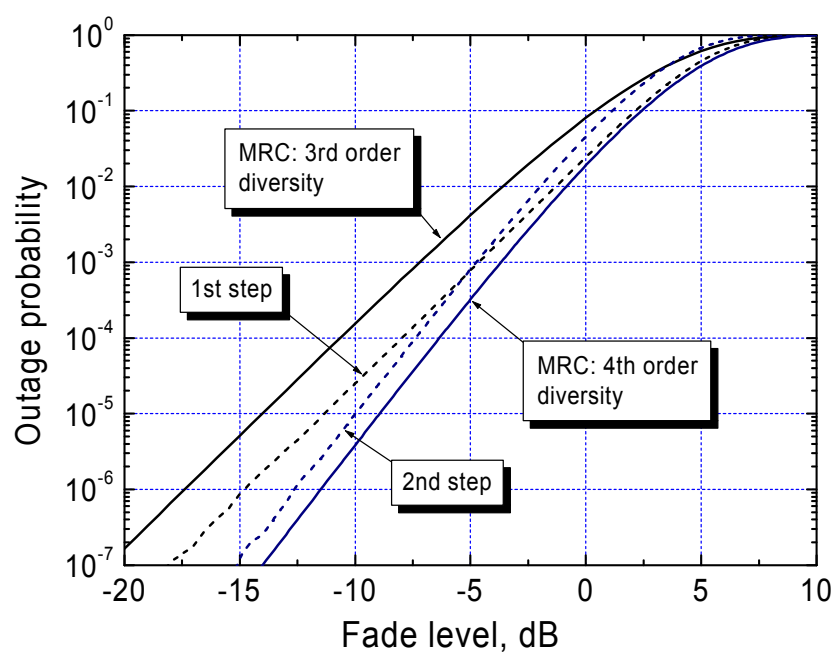

Figure 5. Outage probability curves of the V-BLAST algorithm for $\mathrm{n}=4$.

at $i$-th processing step is $(n-m+i)$, provided that no optimal ordering is used.
Numerical Monte-Carlo simulations validate the analytical results above and allow us to analyze the BLAST performance with the optimal ordering. The effect of the optimal ordering for a moderate number of antennas is approximately to increase the after processing SNR rather than to increase the diversity order. The same is true for a larger number of antennas and lower outage probabilities.

Unfortunately, we are not able at the moment to analyze analytically the outage probability of the V-BLAST with optimal ordering due to the absence of an appropriate mathematical technique. Order statistics are usually employed for a diversity combining (or space-time coding with full diversity) analysis in a similar situation. However, this approach does not apply directly to the V-BLAST with optimal ordering due to higher dimensionality of the problem. Hence, some extension of the order statistics approach is required to analyze analytically the optimal ordering V-BLAST. In the present paper, we employed a numerical Monte-Carlo approach to this problem.

\section{REFERENCES}

[1] G.J. Foschini, M.J. Gans, On Limits of Wireless Communications in a Fading Environment when Using Multiple Antennas, Wireless Personal Communications, vol. 6, No. 3, pp. 311-335, March 1998.

[2] G.J Foschini, 'Layered space-time architecture for wireless communication in a fading environment when using multiple antennas', Bell Lab. Tech. J., vol. 1, N. 2, pp. 41-59, 1996.

[3] I.E. Telatar, "Capacity of Multi-Antenna Gaussian Channels," AT\&T Bell Lab. Internal Tech. Memo., June 1995 (European Trans. Telecom., v.10, N.6, Dec.1999)

[4] G.G. Rayleigh, J.M. Cioffi, Spatio-Temporal Coding for Wireless Communications, IEEE Trans. Commun., v.46, N.3, pp. 357-366, 1998.

[5] G.D. Golden, G.J. Foschini, R.A. Valenzuela, P.W. Wolniansky, 'Detection Algorithm and Initial Laboratory Results Using V-BLAST Space-Time Communication Architecture', Electronics Letters, vol. 35, No. 1, pp.14-16, $7^{\text {th }}$ January 1999.

[6] S.L. Loyka, J.R. Mosig, Channel Capacity of N-Antenna BLAST Architecture, Electronics Letters, vol. 36, No.7, pp. 660-661, Mar. 2000.

[7] S.L. Loyka, Channel Capacity of MIMO Architecture Using the Exponential Correlation Matrix, IEEE Communicatons Letters, v.5, N. 9, pp. $369-371$, Sept. 2001

[8] S. Loyka, A. Kouki, The Impact of Correlation on Multi-Antenna System Performance: Correlation Matrix Approach, 2001 IEEE Vehicular Technology Conference, Atlantic City, USA, Oct. 7-11, pp. 533-537.

[9] G.J Foschini et al, Simplified Processing for High Spectral Efficiency Wireless Communication Employing Multi-Element Arrays, IEEE Journal on Selected Areas in Communications, v. 17, N. 11, pp. 1841-1852, Nov. 1999.

[10] F.R. Gantmaher, Theory of Matrices, Nauka, Moscow, 1988 (in Russian).

[11] S.L. Loyka, Channel Capacity of Two-Antenna BLAST Architecture, Electronics Letters, vol. 35, No. 17, pp. 1421-1422, 19th Aug. 1999.

\section{APPENDIX}

A closed-form analytical model of the Gramm-Schmidt process is given in [10, pp. 213-221]. Let us consider the i-st processing step. Assuming that the first $(i-1)$ symbols are detected without errors and the interference cancellation is accomplished, the received vector at this step is: 


$$
\mathbf{r}_{i}{ }^{\prime}=\mathbf{r}-\sum_{k=1}^{i-1} \mathbf{h}_{k} q_{k}=\sum_{k=i}^{m} \mathbf{h}_{k} q_{k}
$$

The orthogonal projection of $\mathbf{r}_{i}{ }^{\prime}$ into the space spanned by $\left\{\begin{array}{lll}\eta_{i+1} & \ldots & \eta_{m}\end{array}\right\}$ is given by [10, p. 214]:

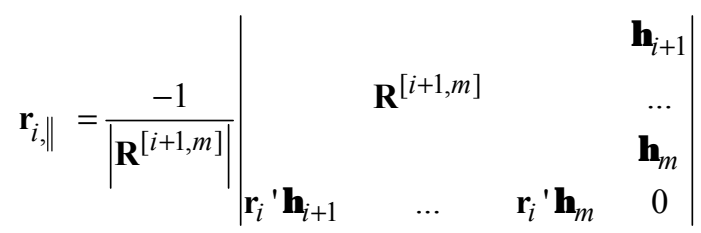

The component of $\mathbf{r}_{i}{ }^{\prime}$ orthogonal to $\left\{\begin{array}{lll}\eta_{i+1} & \ldots & \eta_{m}\end{array}\right\}$ is

$$
\begin{aligned}
& \mathbf{r}_{i, \perp}=\mathbf{r}_{i}{ }^{\prime}-\mathbf{r}_{i, \|}= \\
& \frac{1}{\left|\mathbf{R}^{[i+1, m]}\right|}\left|\begin{array}{cccc} 
& & & \eta_{i+1} \\
& \mathbf{R}^{[i+1, m]} & & \ldots \\
& & & \eta_{m} \\
\mathbf{r}_{i}{ }^{\prime} \eta_{i+1} & \ldots & \mathbf{r}_{i}{ }^{\prime} \eta_{m} & \mathbf{r}_{i}{ }^{\prime}
\end{array}\right|
\end{aligned}
$$

Taking into account (A1), we note that the last row in the numerator determinant in (A3) includes components proportional to 1-st to $(m-i)$-th rows, which can be dropped out because they do no affect the determinant value. Thus, the only component of $\mathbf{r}_{i}{ }^{\prime}$ that gives contribution to the determinant is $\mathbf{h}_{i} q_{i}$. Consequently, (A3) reduces to:

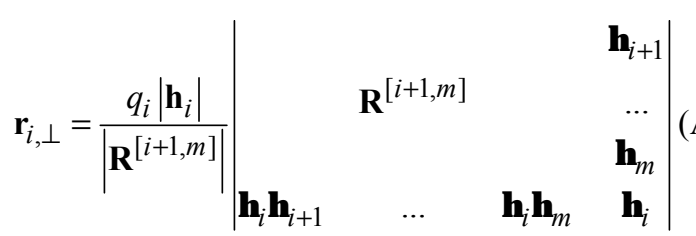

Relocating the last row to the top position and the right column to the left position, one obtains:

$$
\mathbf{r}_{i, \perp}=\frac{q_{i}\left|\mathbf{h}_{i}\right|}{\left|\mathbf{R}^{[i+1, m]}\right|}\left|\begin{array}{cccc}
\eta_{i} & \eta_{i} \eta_{i+1} & \ldots & \eta_{i} \eta_{m} \\
\eta_{i+1} & & & \\
\ldots & & \mathbf{R}^{[i+1, m]} & \\
\eta_{m} & &
\end{array}\right|
$$

The signal power is:

$$
\left|\mathbf{r}_{i, \perp}\right|^{2}=\mathbf{r}_{i, \perp} \mathbf{r}_{i}
$$

Substituting (A6) into (A5), we obtain:

$$
\left|\mathbf{r}_{i, \perp}\right|^{2}=\frac{q_{i}\left|\mathbf{h}_{i}\right|}{\left|\mathbf{R}^{[i+1, m]}\right|}\left|\begin{array}{cccc}
\eta_{i} \mathbf{r}_{i} & \eta_{i} \eta_{i+1} & \ldots & \eta_{i} \eta_{m} \\
\eta_{i+1} \mathbf{r}_{i} & & & \\
\ldots & & \mathbf{R}^{[i+1, m]} & \\
\eta_{m} \mathbf{r}_{i} & &
\end{array}\right|
$$

We note that the first column in the numerator determinant in (A7) includes components proportional to 2-nd to ( $m$ $i+1)$-th columns, which can be dropped out. Consequently, (A7) reduces to:

$$
\begin{aligned}
& \left|\mathbf{r}_{i, \perp}\right|^{2}=\frac{\left|q_{i}\right|^{2}\left|\mathbf{h}_{i}\right|^{2}}{\left|\mathbf{R}^{[i+1, m]}\right|}\left|\begin{array}{cccc}
1 & \eta_{i} \eta_{i+1} & \ldots & \eta_{i} \eta_{m} \\
\eta_{i+1} \eta_{i} & & & \\
\ldots & & \mathbf{R}^{[i+1, m]} & \\
\eta_{m} \eta_{i} &
\end{array}\right|= \\
& \left|q_{i}\right|^{2}\left|\mathbf{h}_{i}\right|^{2} \frac{\left|\mathbf{R}^{[i, m]}\right|}{\left|\mathbf{R}^{[i+1, m]}\right|}
\end{aligned}
$$

